\title{
Thermally and optically tunable sub-terahertz superconducting fishnet metamaterial
}

\author{
Cumali Sabaha a,b,*, Batuhan Mullac ${ }^{c}$, Hakan Altan ${ }^{\mathrm{d}}$, Lutfi Ozyuzer ${ }^{\mathrm{e}}$ \\ a Department of Electrical and Electronics Engineering, Middle East Technical University-Northern Cyprus Campus (METU-NCC), Kalkanli, TRNC/Mersin 10, \\ Guzelyurt 99738, Turkey \\ ${ }^{\mathrm{b}}$ Kalkanli Technology Valley, Middle East Technical University-Northern Cyprus Campus (METU-NCC), Kalkanh, TRNC/Mersin 10, Guzelyurt 99738, Turkey \\ ' Sustainable Environment and Energy Systems, Middle East Technical University-Northern Cyprus Campus, Kalkanli, TRNC/Mersin 10, Guzelyurt, Turkey \\ d Department of Physics, Middle East Technical University, Cankaya, Ankara 06800, Turkey \\ e Department of Physics, Izmir Institute of Technology, Urla, Izmir 35430, Turkey
}

\section{A R T I C L E I N F O}

\section{Article history:}

Received 14 February 2017

Revised 5 September 2017

Accepted 20 October 2017

Available online 13 November 2017

\section{Keywords:}

Metamaterial

Superconductivity

Terahertz

Thermal tunability

Optical tunability

\begin{abstract}
A B S T R A C T
In this paper, a novel fishnet metamaterial structure is designed and analyzed under different material combinations and under different active controlling techniques. The results indicate that, the proposed fishnet metamaterial has a single resonance with double negativity at $0.39 \mathrm{THz}$ when quartz substrate and aluminum is utilized in the design. Moreover, when the metallic parts are replaced with YBCO, the proposed design also exhibits double negativity with a stronger resonance and can be used as a switch between the double negative and single negative modes if the temperature is altered. In addition to these, when substrate (quartz) is replaced with $\mathrm{MgO}$, the resonance shifts from $0.39 \mathrm{THz}$ to $0.26 \mathrm{THz}$ and shows double negativity. Moreover, switching properties under illumination can also be obtained when the silicon is utilized in the design (MgO-YBCO combination). According to these results, it is found that, in the case that the conductivity of silicon exceeds a certain value, the character of the resonance changes from double negative to the single negative mode.
\end{abstract}

(c) 2017 Elsevier B.V. All rights reserved.

\section{Introduction}

Most natural materials do not have a strong response in the low frequency terahertz region where applications in sensing imaging and communication are continuously gaining importance [17]. To overcome this limitation, subwavelength, artificially engineered materials, metamaterials, can be utilized in the so called $\mathrm{THz}$ gap. Metamaterials can simultaneously exhibit negative permittivity and negative permeability. Simultaneous presence of both the negative permittivity and negative permeability have gained significant attention due to the exotic electromagnetic properties that these materials exhibit [8].

Electromagnetic characteristics of the metamaterials can be tailored by scaling the unit structure of these materials [9]. Therefore, the geometric dimensions can be arranged so that a metamaterial can give a response in the low frequency terahertz region for a desired application. Although the response can change by scaling the geometric dimensions, it is not practical and requires fabrica-

\footnotetext{
* Corresponding author at: Department of Electrical and Electronics Engineering, Middle East Technical University-Northern Cyprus Campus (METU-NCC), Kalkanli, TRNC/Mersin 10, Guzelyurt 99738, Turkey.

E-mail address: sabah@metu.edu.tr (C. Sabah).
}

tion of different samples for different applications. However, when the metamaterials are combined with the reconfigurable elements, electromagnetic response can be controlled by just changing the environment instead of changing the dimensions. Hence the active control of the metamaterial can be realized. The reconfigurable elements for active control of the metamaterial can be semiconductors [10], graphene [11], superconductors [12], etc. When an external stimulation is applied to these materials, such as temperature, optical pump, and voltage control, their properties can be altered [1].

In metamaterial structures, generally, conductive elements are made up of metals or semiconductors [13]. With the integration of a photoconductive semiconductor around the metallic structure, active control of the metamaterial is possible. When an external pump beam is applied, changes in the conductivity of the photo conducting semiconductor results in a change in the capacitance of the structure, and therefore a shift in the resonance frequency [14].

In addition to the photo conductive semiconductor materials used in the metamaterial structures, the active controlling can also be done by replacing the metallic parts with superconductors. In most applications of metamaterials, metals are being utilized in the conductive structure. However, the utilization of superconduc- 


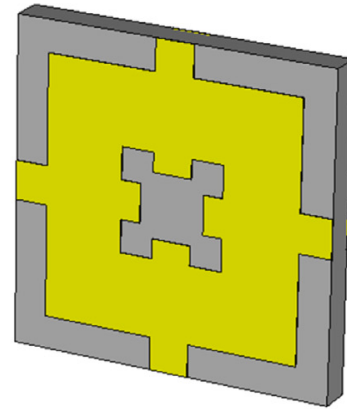

(a)

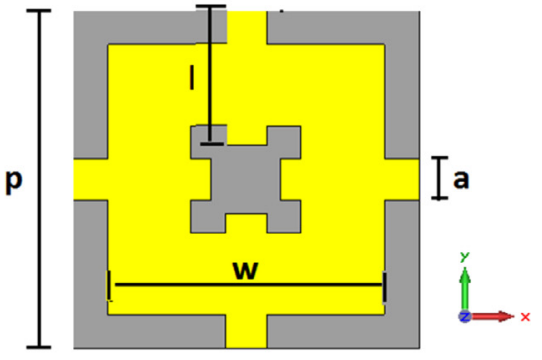

(b)

Fig. 1. Geometry of the unit cell of the proposed fishnet metamaterial structure. (a) Perspective view (b) Front view.

tors as the conductive elements is gaining interest [15]. In contrast to metals, the resistance of the superconductors strongly depends on temperature, magnetic field and applied optical fields [16,17]. Moreover, under some appropriate conditions, the ohmic loss of the superconducting material may be reduced to zero [16]. Therefore, by introducing superconductor materials into the metamaterial structures, the direct control of the metamaterial can be possible by changing the temperature which results in a change in the conductivity.

In this paper, a novel fishnet metamaterial design consisting of a dielectric layer sandwiched between the two metallic dielectric layers which provides negative permittivity and negative permeability is introduced and analyzed with numerical simulations. The characterization of the proposed fishnet metamaterial is carried out for different dielectric and metallic layers. In order to turn the designed structure from being a passive metamaterial into an active metamaterial, the metallic layers are replaced with superconductor and/or semiconducting layers. Under this condition, double negativity property of the designed structure is investigated. Moreover, the proposed superconductor based design is also analyzed with respect to its temperature dependence while the semiconductor based layer is analyzed under external illumination.

\section{Design and simulation}

A schematic representation of the proposed fishnet metamaterial with the relevant geometrical dimensional parameters is shown in Fig. 1. The structure is composed of two metallic plates (yellow parts in Fig. 1) and a dielectric substrate sandwiched between the metallic plates. The metallic resonators are composed of the four strips embedded in the square ring as shown in Fig. 1a.

The unit cell periodicity of the proposed fishnet metamaterial structure is given by the parameter " $\mathrm{x}$ " where the dimensions of the metallic square ring resonators are expressed by the parameter "a". The values associated to the geometric parameters shown in Fig. $1 \mathrm{~b}$ are $p=250 \mu \mathrm{m}, w=200 \mu \mathrm{m}, a=30 \mu \mathrm{m}$, and $l=100 \mu \mathrm{m}$. The thickness of the metallic parts and the thickness of the dielectric substrate are $1 \mu \mathrm{m}$ and $25 \mu \mathrm{m}$, respectively.

The numerical simulation of the superconducting fishnet metamaterial is performed by a full wave electromagnetic wave simulation based on finite integration technique. The frequency range for the numerical study is chosen to be sub-terahertz. Periodic boundary conditions are employed along the lateral directions while waveguide ports are used for the excitations and detection of the sub-THz wave in the z-direction.

\section{Results and discussion}

In this paper, numerical simulations for the characterization of the designed structure were performed according to five different dielectric-metal combinations. For all of the material combinations, the magnitudes of the S-parameters as well as effective permittivity and the permeability of the proposed fishnet metamaterial are analyzed and presented. Initially, aluminum was employed for the metallization, while quartz was used as the dielectric substrate. Then, an active fishnet metamaterial was obtained by replacing the aluminum parts with a high temperature superconductor material, yttrium barium copper oxide (YBCO). As the conductivity of YBCO is strongly dependent on the temperature, the response of the proposed fishnet metamaterial was analyzed under different temperatures. Afterward, quartz is replaced with magnesium oxide $(\mathrm{MgO})$ as dielectric substrate, whose dielectric permittivity is almost stable under low temperatures. Finally, the inner gaps of the metallic parts of the final structure were filled with a semiconductor material, silicon ( $\mathrm{Si}$ ), and studied when an external pump beam is applied to vary its conductivity.

\subsection{Quartz-aluminum combination}

Aluminum with electrical conductivity of $3.57 \times 10^{7} \mathrm{~S} / \mathrm{m}$ and quartz with $\varepsilon=4.45$ and loss tangent of 0.00019 [18] were used as the metallic structure and for the dielectric substrate respectively. The simulation result for S-parameters (for magnitude see Fig. 2a and for phase see Fig. 2b) are shown in Fig. 2. The reflection minima, transmission peak and phase changes occur at around $0.39 \mathrm{THz}$ with a transmission magnitude of 0.71 . In order to characterize the resonance, effective parameters (permittivity $(\varepsilon)$ and permeability $(\mu)$ ) are extracted. The frequency dependent response of the extracted effective parameters is given in Fig. 3 (Fig. 3a being the effective permittivity and Fig. $3 b$ being the effective permeability). The $\mathrm{THz}$ response of the structure shows a Drude-like electrical behavior in the permittivity, while it has a Lorentz-like magnetic behavior in the permeability. It means that the dielectric and magnetic constants of the structure under the THz illumination displays dispersive Drude-like and Lorentz-like responses, respectively. For this reason, both the real part of $\varepsilon$ and the real part of $\mu$ will be negative around the resonant frequency confirming the double negativity for this resonance, as seen from Fig. 3. The magnitude of the $\varepsilon$ and $\mu$ are -0.29 and -0.082 at around $0.39 \mathrm{THz}$, in order.

The current distributions on the metallic parts (front and back) at the resonant frequency are shown in Fig. 4. The anti-symmetric response of the surface current distributions (around the upper and lower parts of the structure) corresponds to the magnetic resonance. The displacement current circulates between the plates and closes the current loop. Therefore, a magnetic field is induced as a result of the circulating current. Hence, the induced response (diamagnetism) produces strong resonances which yield as a negative permeability given in Fig. 3. The surface current distributions given 


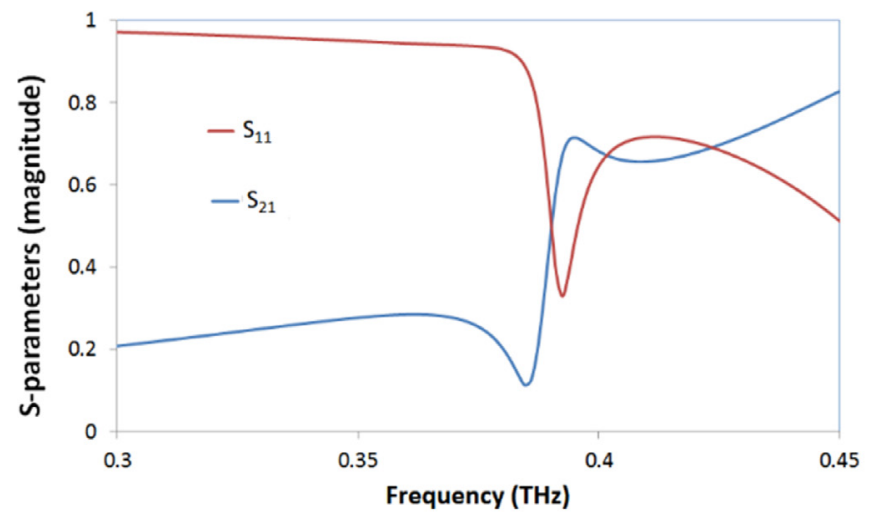

(a)

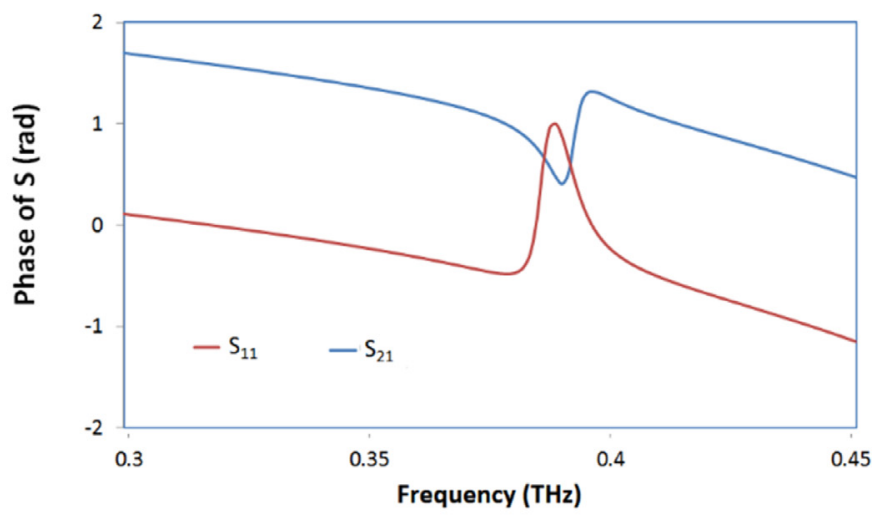

(b)

Fig. 2. Frequency response of the reflection and transmission for the proposed fishnet metamaterial structure when the combination of quartz and aluminum are used.

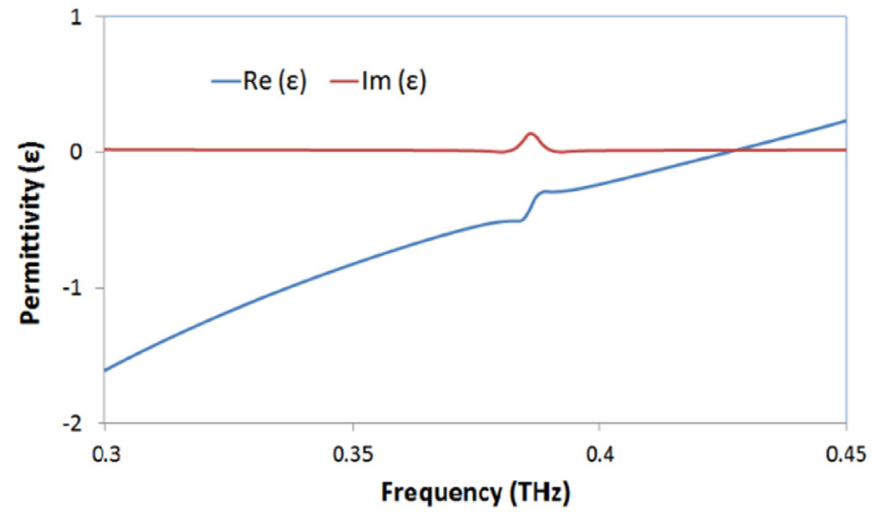

(a)

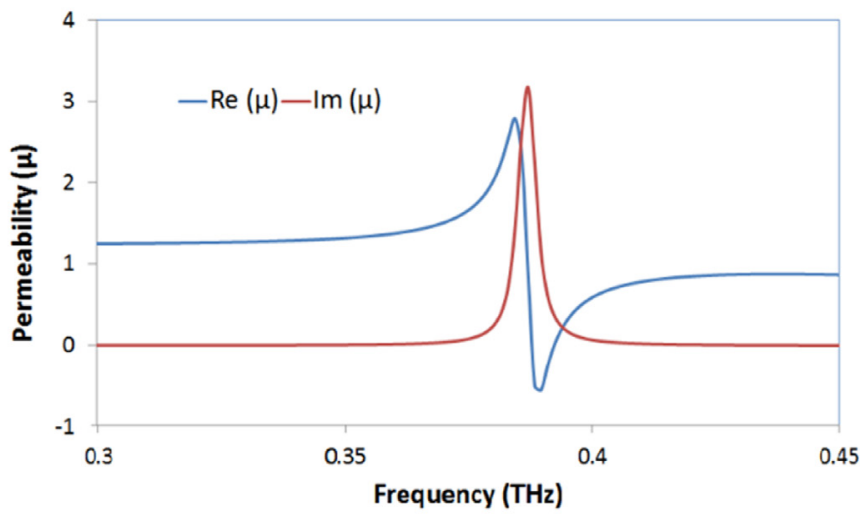

(b)

Fig. 3. Extracted effective parameters retrieved from the reflection and transmission data (a) effective permittivity (b) effective permeability.

Front

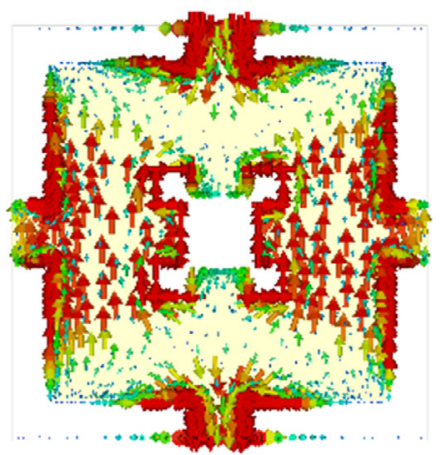

Back

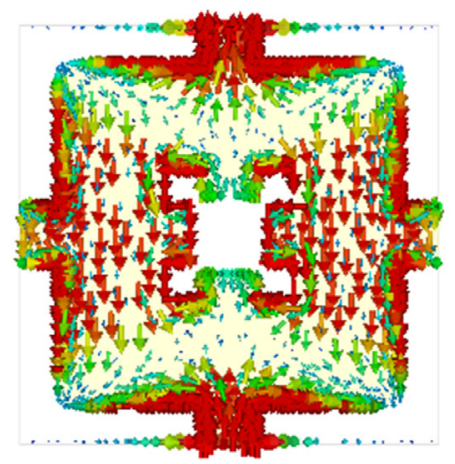

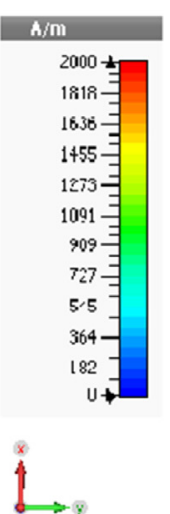

Fig. 4. Current distribution of the proposed structure at the resonance frequency.

here are consistent with similar structures studied in the literature [19-24].

\subsection{Quartz-YBCO combination}

Here, aluminum parts are replaced with a superconductive material, YBCO and quartz was kept as the dielectric substrate. YBCO is a high-temperature superconducting material with a critical temperature of $T_{C}=92 \mathrm{~K}$ [25]. When the superconductor YBCO is employed in the fishnet metamaterial structure, the optical and electromagnetic response of the fishnet metamaterial can be altered by just changing the environmental conditions, i.e. temperature [27]. Therefore, the active controlling of fishnet metamaterial is possible. The electric conductivity of the YBCO material was taken as $1 \times 10^{10} \mathrm{~S} / \mathrm{m}$ [12]. The results for the reflection, transmission and phase changes are given in Fig. 5. The resonance frequency occurs at around $0.39 \mathrm{THz}$ with a transmission magnitude of 0.97 . When compared to the resonance in the previous part, the strength of the resonance is enhanced. This is mainly because of the differences in the conductivity of the metallic struc- 


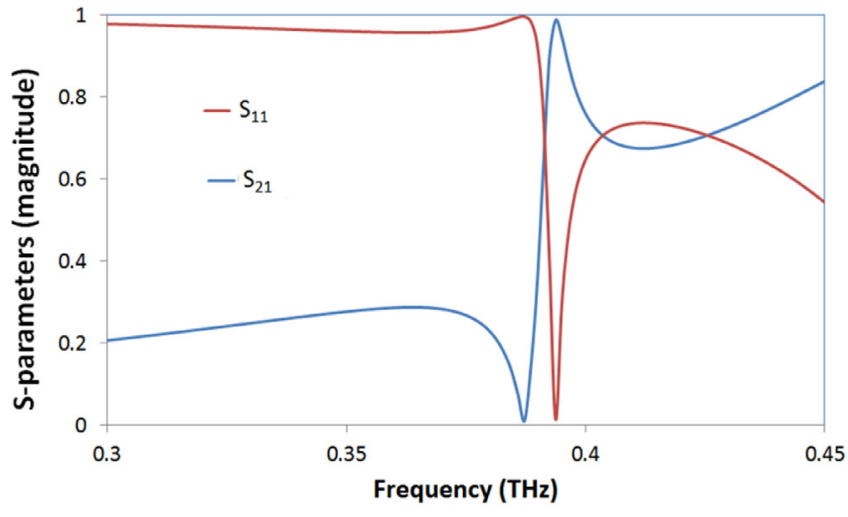

(a)

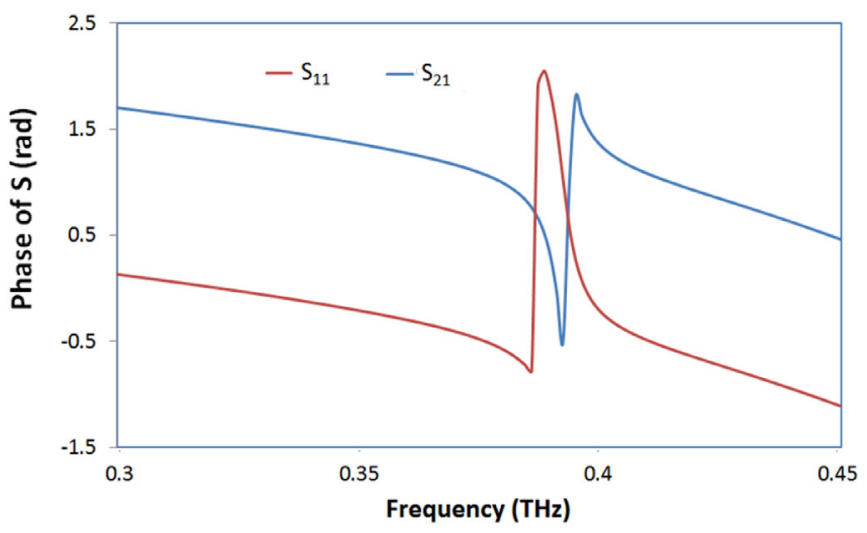

(b)

Fig. 5. Frequency response of the reflection and transmission for the proposed fishnet metamaterial structure when the combination of quartz and YBCO are used.

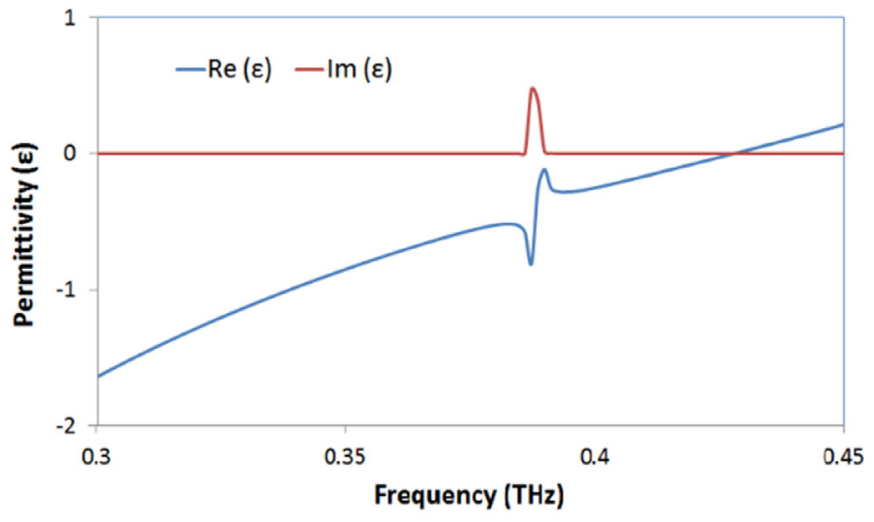

(a)

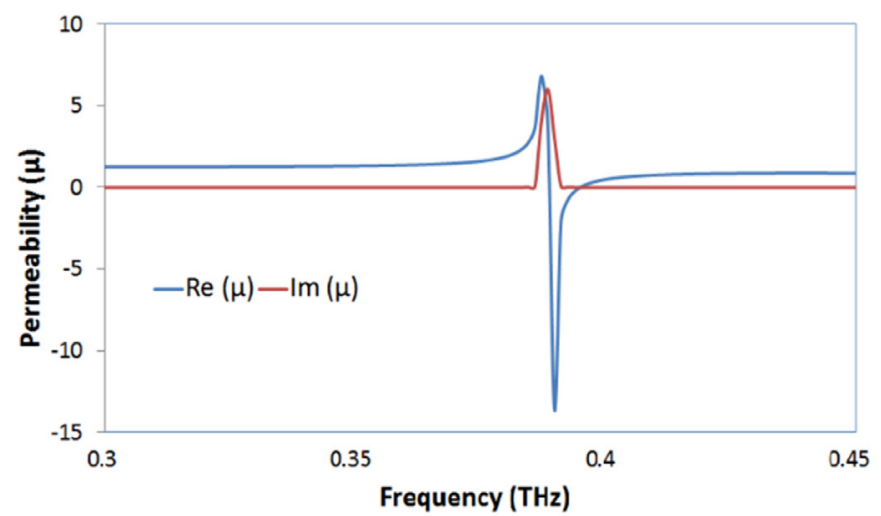

(b)

Fig. 6. Extracted effective parameters retrieved from the reflection and transmission data (a) effective permittivity (b) effective permeability.

ture. Aluminum has a conductivity of the order of $10^{7}$ while YBCO has $10^{10} \mathrm{~S} / \mathrm{m}$. When the conductivity becomes greater, the electric and magnetic resonance becomes stronger which results in the enhancement of the transmission and reduction in reflection.

The frequency dependent response of the extracted effective permittivity and effective permeability are given in Fig. 6a and b respectively. At the resonance frequency, $\varepsilon$ has the value of -0.28 and $\mu$ has the value of -0.66 . As can be clearly seen from these figures, the effective parameters are stronger when compared to the previous material combination. Therefore, as discussed above, the structure has a double negativity around the resonance frequency with an enhanced magnitude.

In addition, the surface current distributions for this combination are also provided in Fig. 7. Here the distributions show similar characteristics with Fig. 4. However, the magnetic response caused by the eddy current is relatively stronger when compared with Fig. 4. This stronger magnetic resonance causes a deeper negative permittivity (Fig. 6).

\subsection{Quartz-YBCO combination with varying temperature (Thermal tunability)}

As the conductivity of YBCO is strongly dependent on the temperature, in this part, response of the proposed fishnet metamaterial was analyzed under different temperatures. For the numerical analysis, quartz was modeled with a constant $\varepsilon=4.45$ and loss of the tangent of 0.00019 and the two-fluid model was used to introduce the temperature dependent conductivity of YBCO in the simu- lation $[25,26]$. For modeling purposes, the values of the parameters were taken from [27]. The S parameter reflection and transmission results of the simulation are given in Fig. 8a and b respectively. As it is clear from these figures, when the temperature is increased the strength of the resonance starts to decrease. This is mainly because of the changes in the conductivity of YBCO when the temperature is altered.

Moreover, the temperature dependent frequency response of the extracted effective permittivity and effective permeability are given in Fig. 9a and b respectively. As the temperature increases the effective parameters, especially permeability, tend to increase. The real part of the permittivity is negative at the resonance frequency. However, the real part of the permeability is only negative when the temperature is below $50 \mathrm{~K}$. After $50 \mathrm{~K}$, the double negativity property of the fishnet metamaterial is lost because of the loss in magnetic resonance. Therefore, after this temperature, resonance has turned into a single negative character.

\section{4. $\mathrm{MgO}-\mathrm{YBCO}$ combination}

For this task, quartz is replaced with a magnesium oxide (MgO) substrate, whose dielectric permittivity is almost stable at low temperatures. The dielectric permittivity of $\mathrm{MgO}$ substrate is taken as $\varepsilon=9.7$ [28] where YBCO is modeled with a fixed conductivity of $1 \times 10^{10} \mathrm{~S} / \mathrm{m}$. The numerical result for S-parameters for both magnitude (Fig. 10a) and phase (Fig. 10b) are given in Fig. 10. The reflection minima, transmission peak and phase changes occur at around $0.26 \mathrm{THz}$ with a transmission magnitude of 0.87 . Based on 
Front

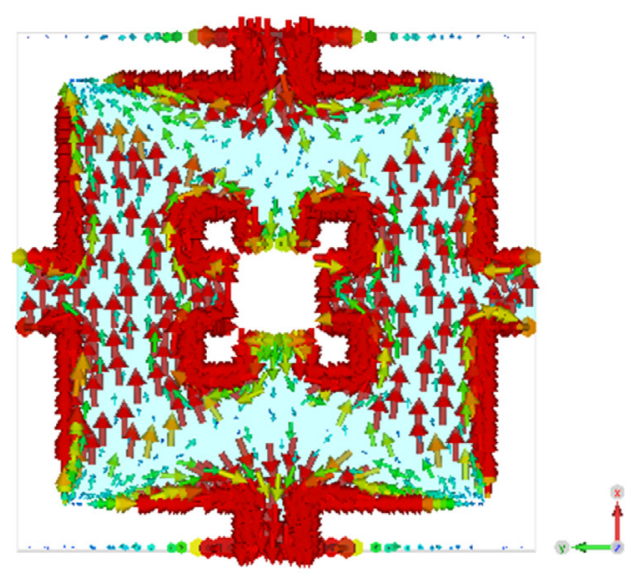

Back

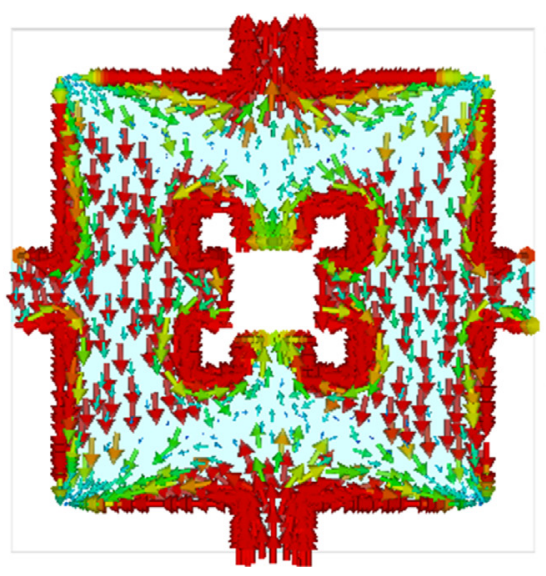

$\mathrm{A} / \mathrm{m}$

2000 当

1818 三

1455 手

1273 手

1091

727 三

545

182 手

$182 \exists$

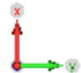

Fig. 7. Current distribution of the proposed structure at the resonance frequency.

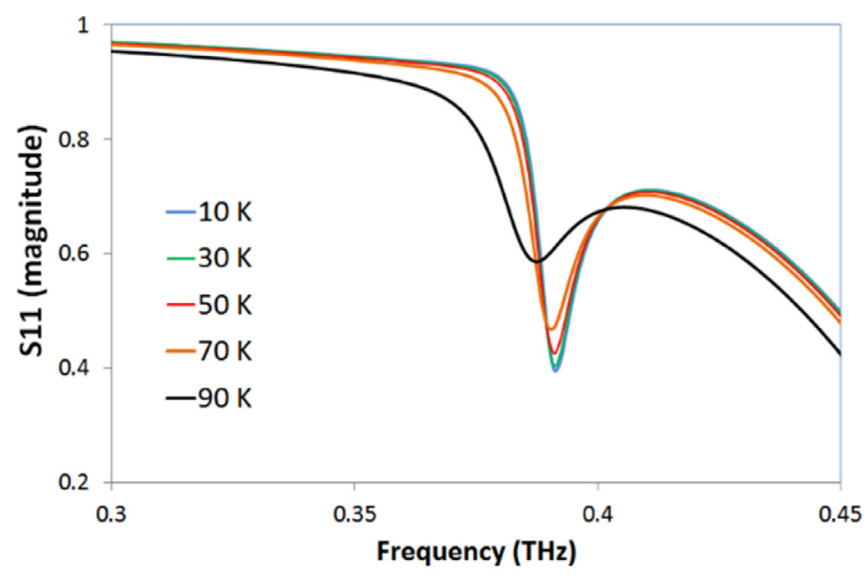

(a)

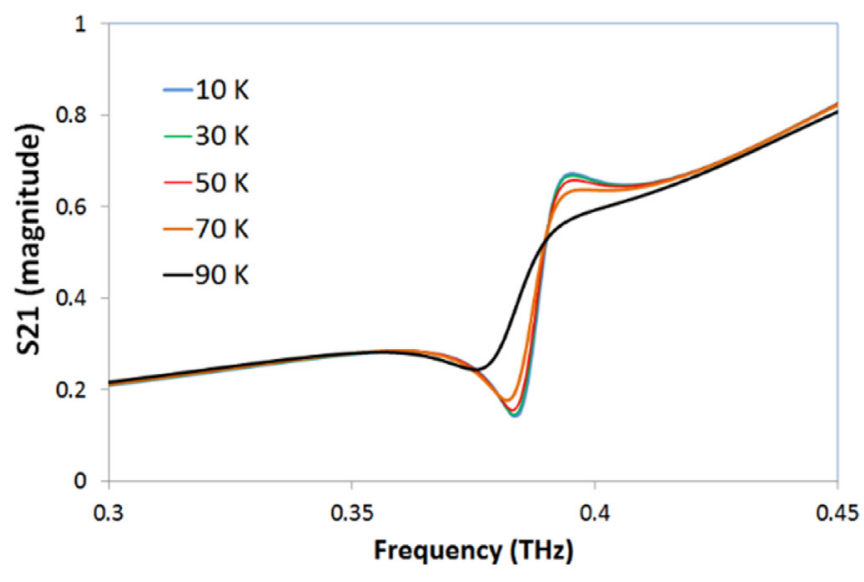

(b)

Fig. 8. Frequency response of the reflection and transmission for the proposed fishnet metamaterial structure when the temperature is altered.

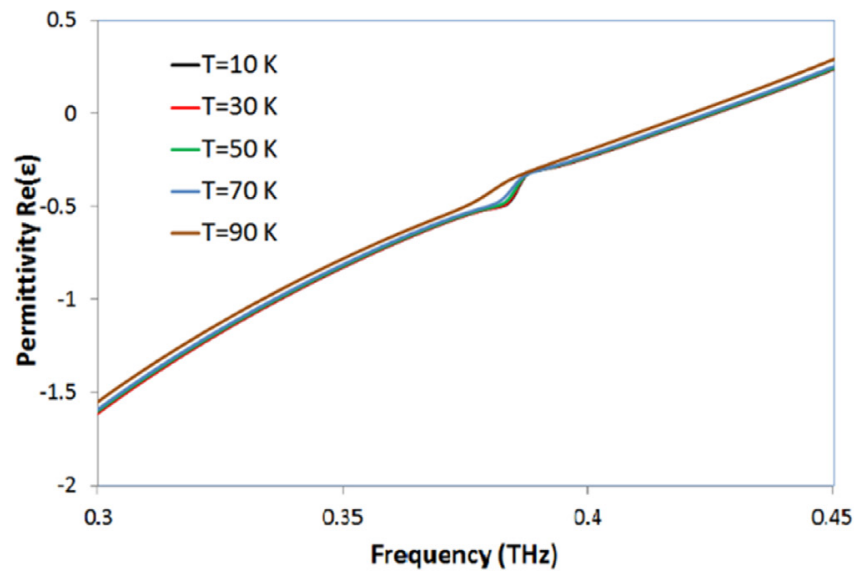

(a)

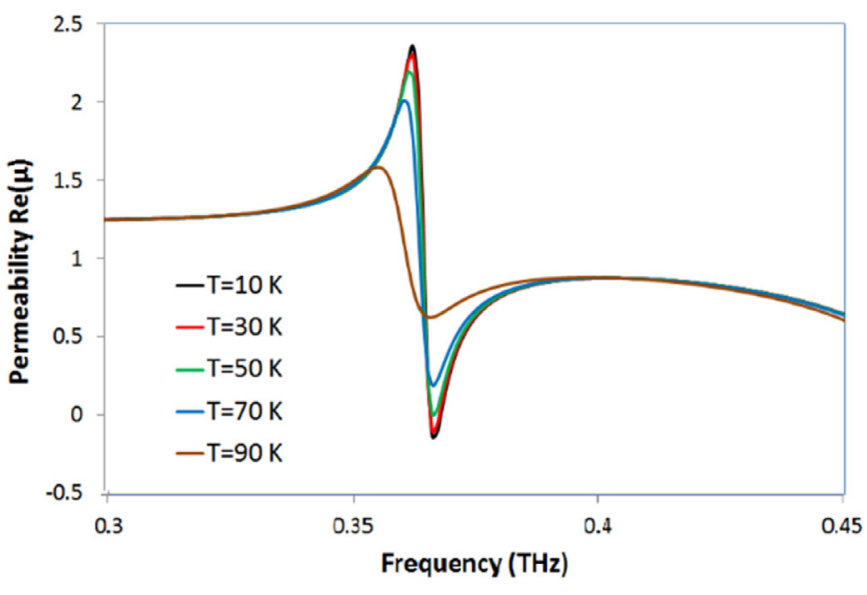

(b)

Fig. 9. Extracted effective parameters retrieved from the reflection and transmission data (a) effective permittivity (b) effective permeability. 


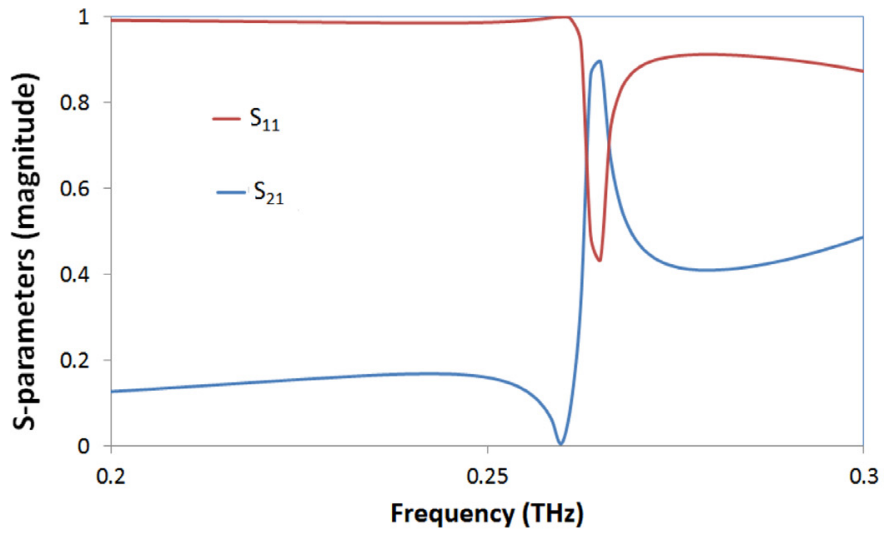

(a)

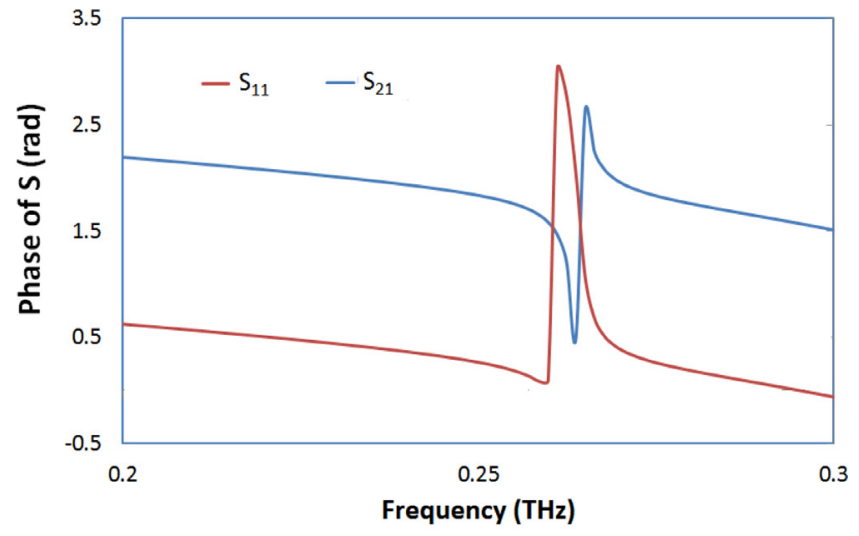

(b)

Fig. 10. Frequency response of the reflection and transmission for the proposed fishnet metamaterial structure when the combination of MgO and YBCO are used.

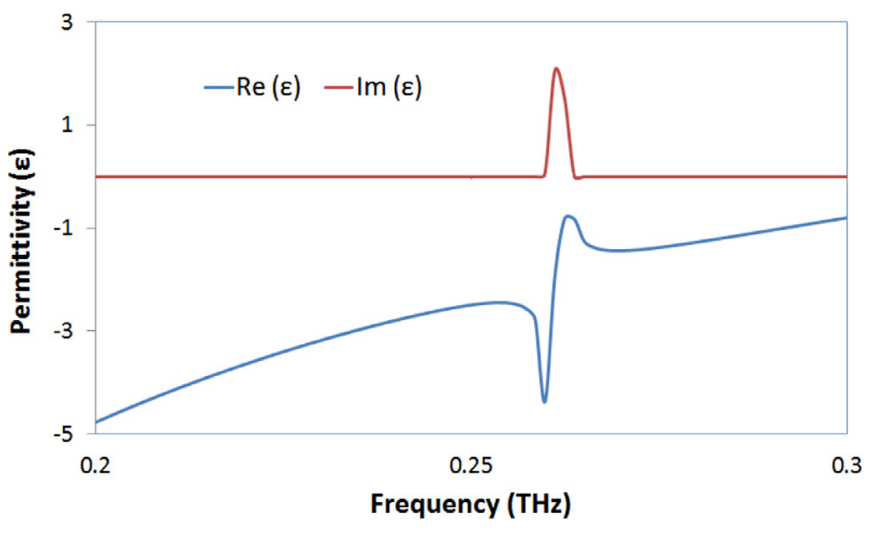

(a)

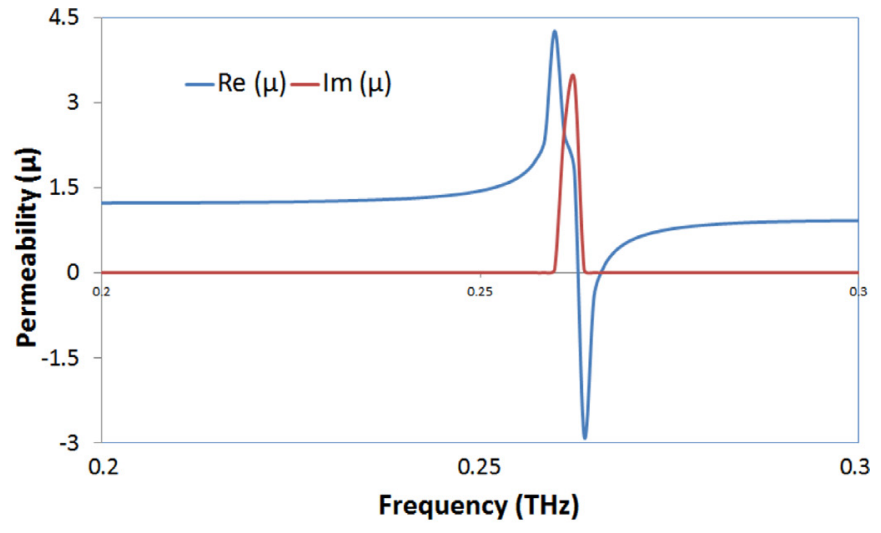

(b)

Fig. 11. Extracted effective parameters retrieved from the reflection and transmission data (a) effective permittivity (b) effective permeability.
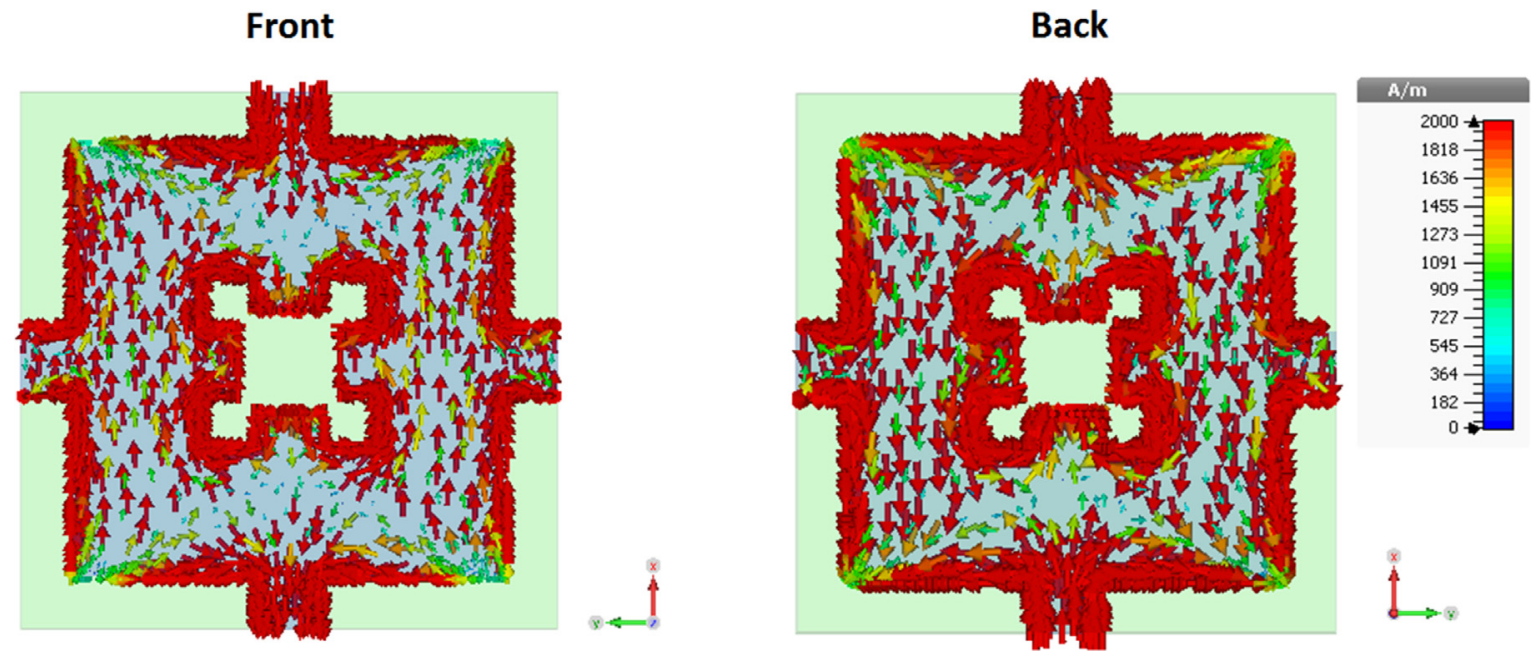

Fig. 12. Current distribution of the proposed structure at the resonance frequency.

the simulation results, it can be concluded that the fishnet metamaterial properties is strongly affected from the change in the substrate. Changing the substrate from quartz to $\mathrm{MgO}$ which increased the dielectric constant (from 4.45 to 9.7), results in a frequency shift of the resonance toward lower frequencies.

The spectra for the extracted effective parameters (see Fig. 11a for effective permittivity and Fig. 11b for effective permeability) are given in Fig. 11. At the resonance frequency, the real part of $\varepsilon$ has the value of -0.89 where the real part of $\mu$ has the value of -2.45 . It is clear that changing the substrate enhanced the magnitude of the both $\varepsilon$ and $\mu$ which leads to a stronger double negativity around the resonance frequency.

Fig. 12 represents the surface current distributions at resonant frequency for MgO-YBCO combination. The distributions show 


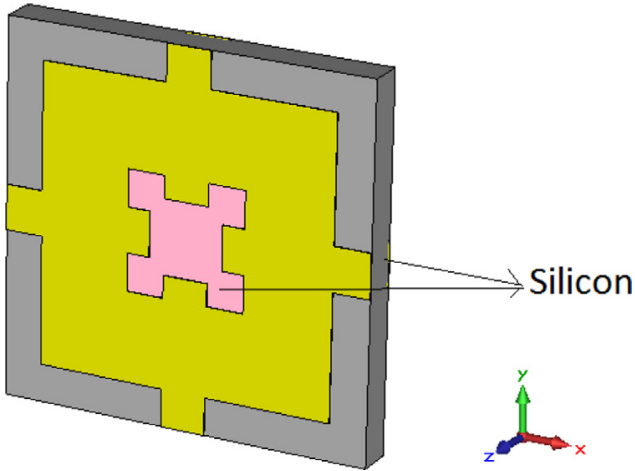

Fig. 13. Representation of the structure when the inner gaps of the resonator are filled with silicon.

similar results with Figs. 4 and 7 . Changing the substrate from quartz to $\mathrm{MgO}$, affected the magnetic response causing the relatively weaker current density when compared to Fig. 7. Moreover, due to the reduced magnetic response, the strength of the permeability is also reduced as shown in Fig. 11b.

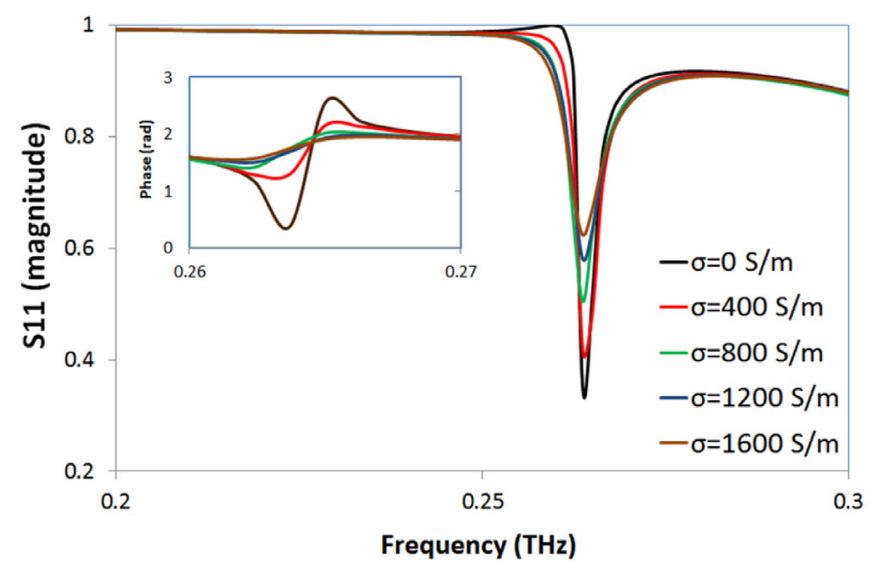

(a)

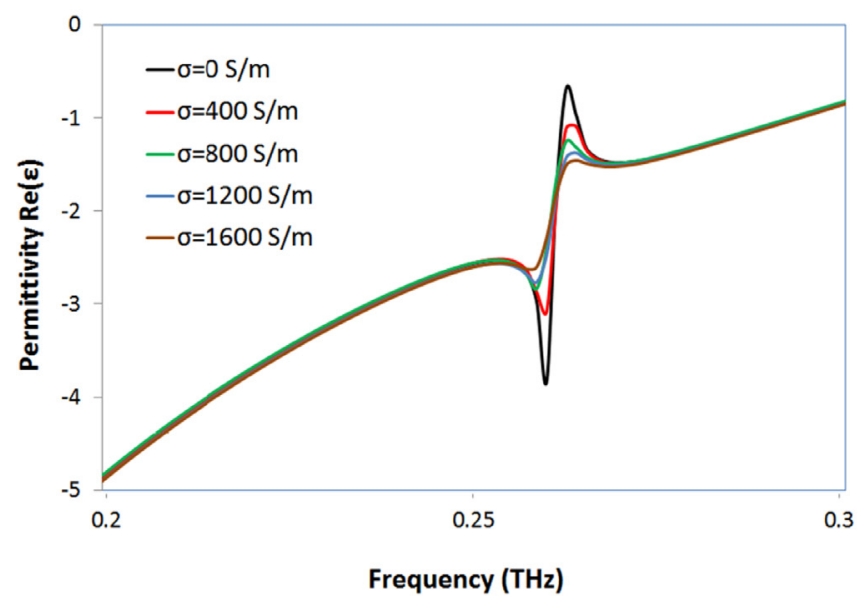

(c)

\subsection{MgO-YBCO combination with external pump beam (Optical tunability)}

The last investigation was to implement a photoconductive semiconductor material (Silicon) into the inner gaps of the resonators as shown in Fig 13. After the gaps were filled with Silicon (Si), the conductivity of the silicon was altered and the response of the structure was again analyzed. In reality, the conductivity of the silicon can be altered by applying an external pump beam. As the amount of illumination gets higher, the conductivity becomes higher and the conductivity becomes lower when the amount of illumination is reduced. The results for $S$ parameters as well as the real part of the permittivity and permeability are shown in Fig. 14. The magnified phase results at the resonant frequency are also given in the inset of Fig. 14a and b.

It can be seen from Fig. 14a and b that the external illumination and the strength of the resonance are inversely proportional. Applying a higher amount of illumination causes the reduction in the strength of the resonance. For Fig. 14, $\sigma=0 \mathrm{~S} / \mathrm{m}$ means that there is no external illumination while $\sigma=1600 \mathrm{~S} / \mathrm{m}$ corresponds to a relatively strong external illumination. In addition, the magnified phase spectra of the corresponding S-parameters are presented in inset of Fig. 14a and b. Fig. 14c and d represents the variation of the permittivity and permeability respectively. The real part of the permittivity is negative at the resonance frequency for

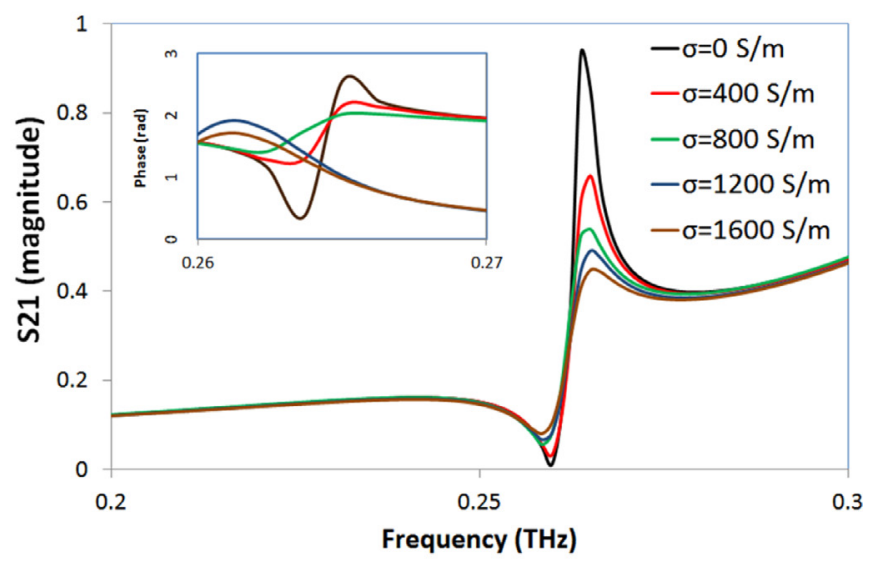

(b)

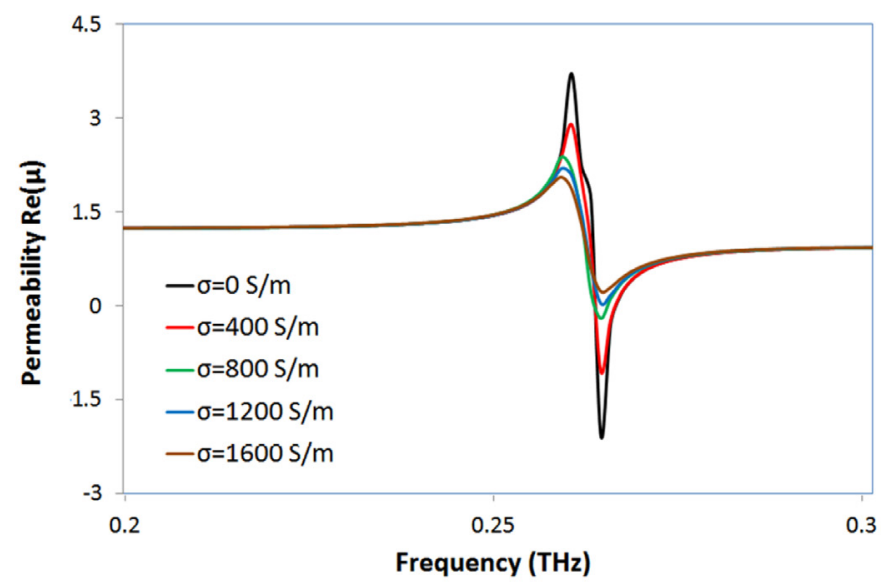

(d)

Fig. 14. Variation of (a) reflection (b) transmission (c) permittivity (d) permeability spectra under different silicon conductivity. 
all the illumination cases. However, the permeability turns positive after $\sigma=1200 \mathrm{~S} / \mathrm{m}$. Therefore, after this point, the resonance turns into single negative mode. Hence we can conclude that, the fishnet metamaterial acts as a switch between double negative and single negative modes.

\section{Discussion of the results}

In this paper, numerical simulations for different dielectricmetal combinations are performed in order to obtain and virtualize the superconductor metamaterial. Usage of superconductors in the structure of metamaterials and formation of metamaterials with superconductors, provide low-loss metamaterials. This is mainly because of the higher conductivity levels of superconductors at low temperatures. Moreover, the superconductor used in this study is YBCO which is known as a high temperature superconductor material. The difference in the $S$ parameters and the double negative character between the aluminum and YBCO are analyzed and the advantage of utilizing YBCO in the design is virtualized. Furthermore, a superconductor metamaterial that operates at low temperatures as well as at relatively higher temperatures is obtained. The double negativity works up to $50 \mathrm{~K}$. In addition to the double negativity and low loss properties of the proposed fishnet metamaterial, switching between double negativity and single negativity is also realized by altering the temperature. The results show that, temperature above $50 \mathrm{~K}$ exhibits the negativity of epsilon.

As it is known, the permittivity of $\mathrm{MgO}$ is almost stable under low temperatures. Therefore, quartz is replaced with $\mathrm{MgO}$ and another switching method for the proposed metamaterial is realized. This method is based on filling the gaps between the metallic parts of the structure with photoconductive silicon. The conductivity of silicon can be altered by the external illumination. The filled silicon negatively effects the electric and magnetic character of the carefully designed YBCO resonator. Thus, even with lower external pump illumination levels, the resonance behavior of the superconductor metamaterial in terms of the $S$ parameters and the double negative behaviors are affected. However, this side effect can be turned into benefit. When the conductivity of Si reaches to $\sigma=1200 \mathrm{~S} / \mathrm{m}$, the permeability turns into positive and the resonance does not show double negative response above this conductivity. Under this condition, switching between single and double negative modes can be realized.

\section{Summary and conclusion}

In conclusion, a novel fishnet metamaterial is designed and analyzed under different material combinations. In addition to the material combinations, fishnet metamaterial is also analyzed under two different active controlling techniques. According to the results, the proposed fishnet metamaterial (with quartz substrate and YBCO superconductor) can be used as a switch between the double negative and single negative modes when the temperature is changed. Moreover, the design can also exhibit switching properties when silicon is utilized in the design. According to these results it is found that under illumination when the conductivity of silicon exceeds $1200 \mathrm{~S} / \mathrm{m}$ the mode changes from a double negative to a single negative mode.

\section{Acknowledgments}

The study reported here was carried out at Middle East Technical University-Northern Cyprus Campus (METU-NCC). It is supported by METU-NCC under the grant numbers of BAP-FEN-15-D-3 and BAP-FEN-16-K-8; and partially supported by TUBITAK (Scientific and Technical Research Council of Turkey) under the project number of $114 \mathrm{~F} 091$.

\section{References}

[1] J. Cong, Z. Zhou, H. Yao, Y. Fu, N. Ren, Reducing the pump power of optically controlled terahertz metamaterial via tailoring the resistance of the silicon gap region, J. Opt. 17 (2015) 105108.

[2] P.U. Jepsen, D.G. Cooke, M. Koch, Terahertz spectroscopy and imaging - Modern techniques and applications, Laser Photonics Rev. 5 (2011) 124-166.

[3] S. Koenig, D. Lopez-Diaz, J. Antes, F. Boes, R. Henneberger, A. Leuther, A. Tessmann, R. Schmogrow, D. Hillerkuss, R. Palmer, T. Zwick, C. Koos, W. Freude, O. Ambacher, J. Leuthold, I. Kallfass, Wireless sub-THz communication system with high data rate, Nat. Photonics 7 (2013) 977-981.

[4] Y.D. Sirmaci, C.K. Akin, C. Sabah, Fishnet based metamaterial loaded THz patch antenna, Opt. Quantum Electron. 48 (2016) 168.1-168.10.

[5] Y. Demirhan, M. Kurt, H. Alaboz, T. Semerci, L. Ozyuzer, M.A Nebioglu, T. Takan, H. Altan, C. Sabah, Metal mesh filters based on Ti, ITO and Cu thin films for terahertz waves, Opt. Quantum Electron. 48 (2016) 170.1-170.11.

[6] T. Takan, M.A. Nebioglu, M. Kurt, Y. Demirhan, L. Ozyuzer, C. Sabah, H. Altan, An Indium Tin Oxide Metamaterial Filter for the Terahertz regime: Design, Fabrication and Characterization, 2015 Conference on Lasers and Electro-Optics Europe \& European Quantum Electronics Conference, CLEO/Europe - EQEC 2015, 2015 June 21-25.

[7] Y. Demirhan, H. Alaboz, T. Semerci, L. Ozyuzer, M.A. Nebioglu, T. Takan, H. Altan, C. Sabah, Terahertz wave metametarial filters based on superconducting Bi2212 thin films, Fifth International School and Conference on Photonics-PHOTONICA2015, 2015 August 24-28.

[8] C. Sabah, Microwave response of octagon-shaped parallel plates: Low-loss metamaterial, Opt. Commun. 285 (2012) 4549-4552.

[9] C.M. Watts, X. Liu, W.J. Padilla, Metamaterial electromagnetic wave absorbers, Adv. Mater. 24 (2012) OP98-OP120.

[10] X. Liu, H. Liu, Q. Sun, N. Huang, Metamaterial terahertz switch based on split-ring resonator embedded with photoconductive silicon, Appl. Opt. 54 (2015) 3478-3483.

[11] W.R. Zhu, F. Xiao, M. Kang, D. Sikdar, M. Premaratne, Tunable terahertz left-handed metamaterial based on multi-layer graphene-dielectric composite, Appl. Phys. Lett. 104 (2014) 051902.

[12] F. Trang, H. Rogalla, Z. Popovic, Resonant response of high-temperature superconducting split-ring resonators, IEEE Trans. Appl. Supercond. 23 (2013) 1300405.

[13] B. Heshmat, H. Pahlevaninezhad, T.E. Darcie, Optical efficiency enhancement methods for terahertz receiving photoconductive switches, Opt. Laser Technol. 54 (2013) 297-302.

[14] M. Kafesaki, N.H. Shen, S. Tzortzakis, C.M. Soukoulis, Optically switchable and tunable terahertz metamaterials through photoconductivity, J. Opt. 14 (2012) 114008.

[15] Z. Xiaoling, G. Jianqiang, H. Jiaguang, Z Weili, Tailoring electromagnetic responses in terahertz superconducting metamaterials, Front. Optoelectron. 8 (1) (2015) 44-56

[16] N.K. Grady, B.G. Perkins Jr., H.Y. Hwang, N.C. Brandt, D. Torchinsky, R. Singh, L. Yan, D. Trugman, S.A. Trugman, Q.X. Jia, A.J. Taylor, K.A. Nelson, H.-T. Chen, Nonlinear high-temperature superconducting terahertz metamaterials, New J. Phys. 15 (2013) 105016.

[17] M. Tinkham, Introduction to Superconductivity, second ed., Dover, Mineola, NY, 1996.

[18] E.V. Loewenstein, D.R. Smith, R.L. Morgan, Optical Constants of Far Infrared Materials. 2: Crystalline Solids, Appl. Opt. 12 (1973) 398-406.

[19] A. Yilmaz, C. Sabah, Diamond-shaped hole array in double-layer metal sheets for negative index of refraction, J. Electromagn. Waves Appl. 27 (2013) 413-420.

[20] Z. Huang, J. Xue, Y. Hou, J. Chu, D.H. Zhang, Optical magnetic response from parallel plate metamaterials, Phys. Rev. B 74 (2006) 193105.1-193105.4.

[21] C. Sabah, H.G. Roskos, Numerical and experimental investigation of fishnet-based metamaterial in a X-band waveguide, J. Phys. D Appl. Phys. 44 (2011) 255101.

[22] M. Kafesaki, I. Tsiapa, N. Katsarakis, Th. Koschny, C.M. Soukoulis, E.N. Economou, Left-handed metamaterials: The fishnet structure and its variations, Phys. Rev. B 75 (2007) 235114

[23] C. Sabah, Realization of polarization-angle-independent fishnet-based waveguide metamaterial comprised of octagon shaped resonators with sensor and absorber applications, J Mater Sci: Mater. Electron. 27 (2016) 4777-4787.

[24] C. Sabah, H.G. Roskos, Dual-band polarization-independent sub-terahertz fishnet metamaterial, Curr. Appl. Phys. 12 (2012) 443-450.

[25] O.G. Vendik, I.B. Vendik, D.I. Kaparkov, Empirical model of the microwave properties of high temperature superconductors, IEEE Trans. Microwave Theory 46 (1998) 469-478.

[26] C.-J. Wu, Y.-L. Chen, Microwave properties of a high-temperature superconductor and ferromagnetic bilayer structure, Prog. Electromagn. Res. 111 (2011) 433-445.

[27] H.-T. Chen, H. Yang, R. Singh, J.F. O’Hara, A.K. Azad, S.A. Trugman, Q.X. Jia, A.J. Taylor, Tuning the resonance in high-temperature superconducting terahertz metamaterials, PRL 105 (2010) 247402.

[28] W.-H. Lin, C.-J. Wu, S.-J. Chang, Terahertz intrinsic and effective surface impedances of high-temperature superconducting thin films, J. Electromagn. Waves Appl. 24 (2010) 2589-2603. 